\title{
Les Amours de Psyché et de Cupidon de Jean de La Fontaine comme exemple de prosimètre
}

"J'ai trouvé de plus grandes difficultés dans cet ouvrage qu’en aucun autre qui soit sorti de ma plume $»^{1}(L A: 53)$ - les premiers mots de la Préface de Jean de La Fontaine pour ses Amours de Psyché et de Cupidon laissent juger que le lecteur aura affaire à un ouvrage exceptionnel. Les Amours sont en fait une œuvre hybride, relevant à la fois du conte mythologique, de la pastorale, du poème lyrique, du dialogue philosophique. Réputé aujourd'hui avant tout comme fabuliste, La Fontaine s'est essayé avec plus ou moins de succès à presque tous les genres littéraires. Les trois recueils de Fables alternent avec poèmes, contes, pièces de théâtre, lettres et satires et la fable de La Fontaine semble épouser tous les autres genres. Capricieux, volage, éclectique, inconstant - les épithètes qui caractérisent La Fontaine, homme et artiste, mettent en évidence cette tendance à la diversité. D’autre part, les critiques insistent sur son goût de l'indépendance qui l'empêche de s'asservir et de s'enfermer dans un seul genre littéraire. Pierre Clarac souligne la grande "disponibilité » du poète : "Son instinct d'artiste l'avertissait [...] que le pire danger pour un poète est de se laisser gagner par l'habitude et de devenir le prisonnier de sa propre manière " (1969: 186). Les Amours reflètent admirablement cette propension toute "baroque » du poète et ses multiples talents. Comme le dit Michel Jeanneret, La Fontaine « interroge la géographie des genres littéraires, leurs propriétés, leurs intersections, leurs combinaisons. Psyché participe de cette recherche en rassemblant des matériaux extrêmement divers, afin d’en éprouver la compatibilité » (1991:27).

Les Amours de Psyché et de Cupidon paraissent en 1669 chez Claude Barbin, mais ne connaissent pas le succès attendu. L'ouvre comporte deux livres qui présentent

Dr Alicja Rychlewska-Delimat - maitre de conférences à l'Institut de Lettres et de Langues Modernes de l'Université Pédagogique de Cracovie. Adresse pour correspondance : Instytut Neofilologii, Uniwersytet Pedagogiczny im. KEN, ul. Podchorążych 2, 30-084 Kraków ; e-mail : aliard@op.pl

1. Les références à l'ouvrage analysé de Jean de la Fontaine (Amours de Psyché et de Cupidon) seront désignées par la mention $L A$, suivie du numéro de la page. Nous avons modernisé l'orthographe. 
un curieux, pour l'époque, mélange de genres et de registres, et un amalgame de prose et de vers.

Faisant alterner les passages en prose et les passages versifiés, le texte de La Fontaine peut être considéré comme un prosimètre ${ }^{2}$ et c'est justement en tant que tel que nous voudrions l'analyser. Les dictionnaires définissent le prosimètre comme une forme mixte, prose mêlée de vers ${ }^{3}$. De contenu philosophique, religieux ou moral, ces œuvres prennent souvent une signification allégorique. Les origines du prosimètre remontent à l'Antiquité et le genre reste aussi répandu au Moyen Âge et la Renaissance. Ses premiers exemples - tels les Saturce Menippece de Varron, ou De consolatione philosophice de Boèce - en proposent le modèle stylistique, mais le paradigme du genre semble être fixé dans son plus célèbre exemple - la Vita nova de Dante.

Puisque c'est à ce modèle que nous voudrions nous référer dans notre analyse, rappelons très brièvement les principales caractéristiques de cette œuvre. La Vita nova, qui est une sorte de panégyrique de Béatrice, est qualifiée souvent d' " anthologie amoureuse ». L'ouvrage est un recueil de 31 pièces poétiques, écrites pour la plupart auparavant, que le poète a insérées dans le récit en prose - le tout est enfermé dans 42 chapitres de dimensions brèves. Les passages en prose sont d'un côté le récit autobiographique de l'amour juvénile de Dante pour Béatrice, d'autre part une sorte de glose. Les commentaires que le poète donne de ses vers en expliquent la signification et précisent les circonstances de leur création. Dans une sorte d'introspection, Dante découvre au lecteur toute une gamme de sentiments et toutes les nuances de lamour, amour qui s'impose comme un phénomène mystique. Conformément à la conception stilnoviste, la figure de Béatrice est sublimée, idéalisée et devient une créature parfaite, angélique, quasi-divine.

Mais la matière de l’ouvre ce nest pas uniquement l'histoire de l'amour. Parallèlement à la trame biographique amoureuse, se retrace l'histoire de la vocation et de lévolution poétique de Dante, l'histoire du développement de sa conscience littéraire. La Vita nova peut être considérée comme le texte sur le texte. Certes, c'est Béatrice qui est au centre de l'œuvre, qui en est la source et la substance, mais les vers qu'elle inspire offrent l'occasion à la réflexion toute " technique » sur lécriture elle-même et sur la poétique de la louange (lode) (cf. par ex. le chapitre XXV - un petit traité de la poésie lyrique). Les passages en prose constituent une sorte de cadre qui organise le tout. Les poèmes qui s'inscrivent dans ce tissu en prose sont minutieusement analysés et expliqués par l’auteur. À la manière des razos et des vidas de la tradition troubadouresque provençale, les commentaires en prose apportent l'interprétation des vers à la lumière de la vie du poète. Ces commentaires ont un caractère proprement métatextuel - Dante subdivise ses poèmes en parties, expliquant ses intentions.

Le vers et la prose se correspondent et se complètent dans cette structure bipartite, obéissant à une logique intérieure. De cette tension constante entre le narratif

2. La Fontaine a déjà eu recours à ce genre dans Le Songe de Vaux de 1659.

3. Cf. www.treccani.it. 
et le lyrique se dégage une théorie de la nouvelle poétique, du "style nouveau ». Ainsi, la Vita nova de Dante acquiert en quelque sorte une valeur doctrinale et " programmatique " : tout en faisant adhérer le poète au courant du dolce stil novo, elle lui confère en même temps une touche individuelle.

Quel rapport peut-on voir entre la Vita nova et les Amours de Psyché et de Cupidon de La Fontaine ? Et si nous considérons le texte de Dante comme le paradigme du prosimètre, dans quelle mesure celui de La Fontaine est conforme à un tel modèle ? Entre ces deux ouvrages, apparemment si éloignés et si différents, on trouve une certaine parenté, même si elle n'est sans doute pas intentionnelle ${ }^{4}$. Notre dessein nest pas de faire une analyse comparative des deux œuvres, nous voulons cependant insister sur quelques analogies au niveau de l'histoire, et surtout au niveau de la forme et de l'expression.

Comme chez Dante, le récit du roman de La Fontaine présente une histoire d’amour, on pourrait même dire - d'une quête amoureuse. La Fontaine conte l'amour d'une humaine voué à un dieu, comme l'ouvrage de Dante célèbre une femme dont la figure est divinisée. Dans les deux cas, il s'agit de la douloureuse expérience de l'amour. Sans avancer une lecture allégorique des Amours, notons cependant la portée symbolique de l'histoire de Psyché, dans laquelle Jean Rousset veut voir le «thème platonicien, puis chrétien de lâme en quête du divin » (1976: 122).

Au niveau de la structure, les analogies sont tout aussi manifestes. Les deux ouvres présentent une structure bipartite, mais si dans la Vita nova l'enchâssement comprend précisément les morceaux poétiques, l'architecture des Amours est plus complexe. Dès le premier abord, louvrage de La Fontaine apparaît comme beaucoup plus disparate et d'un plus haut degré d'hybridité. La diversité de sources d'inspiration contribue à renforcer encore l'impression d'hétérogénéité. L'inspiration des Métamorphoses d'Apulée est clairement explicitée par La Fontaine dans sa Préface. La matière ancienne est pourtant traitée "selon la liberté ordinaire qu'[il se] donne » $(L A: 54)$ : " il faut considérer mon ouvrage sans relation à ce qua fait Apulée » (LA: 55), objecte-t-il.

Admirateur de l'Antiquité et fidèle au précepte classique de l'imitation des Anciens, La Fontaine suit assez fidèlement son modèle. Mais d’autres influences paraissent également perceptibles. Premièrement, celle du Décaméron de Boccace. L'histoire de Psyché s'inscrit dans une autre histoire - le récit contemporain de la promenade des quatre amis-poètes dans le parc de Versailles. Ce récit fournit une sorte de cadre aux aventures mythologiques et, à la manière de la cornice boccacienne, organise la narration : au cours de la visite, un des quatre amis, Polyphile, dont les traits renvoient clairement à La Fontaine ${ }^{5}$, lit à ses compagnons le roman qu'il a composé récemment - l'histoire de Psyché selon Apulée. L'auteur introduit dans le récit-cadre

4. Les critiques qui mettent en évidence diverses sources d'inspiration de Psyché ne mentionnent aucune relation avec l'œuvre de Dante.

5. Cf. à ce propos Appendices de l'édition critique des Amours ( $L A: 259)$. 
un groupe d'amis qui forment " une espèce de société " (LA: 59), tout comme les héros du Décaméron. Ce récit constitue, comme chez Boccace, le premier niveau de la narration, avec le narrateur anonyme extradiégétique qui donne la parole à ses acteurs. Mais si les personnages de Boccace racontent, chacun à son tour, plusieurs histoires autonomes, chez La Fontaine, il n'y a qu'un seul narrateur et une seule histoire enchâssée. Au second niveau de la narration, cest Polyphile qui, en narrateur intradiégétique, raconte les aventures de Psyché. Les autres personnages du récit-cadre n'interviennent que rarement, pour commenter le récit de Polyphile, pour exprimer leurs sentiments, etc. Ils auront cependant une voix importante dans le débat sur la poétique qui clôt la première partie de l’œuvre.

Un autre rapprochement qui s'impose, c'est l'influence de l'Astrée d'Honoré d'Urfé. Ce roman pastoral baroque, connu et prisé par tous ses contemporains, nourrit l'imagination de toutes les générations qui suivent. La Fontaine en reprend le cadre bucolique dans lequel il situe la trame sentimentale, ainsi que la conception de l'amour idéalisé. De même, la structure enchâssée de l'Astrée et l'insertion de la poésie au sein du récit l'apparentent à l'ouvrage de La Fontaine.

Les critiques notent également l'influence du Songe de Poliphile de Francesco Colonna et du poème Adone de Marino. On peut voir enfin dans les Amours l'écho des dialogues de Platon, non seulement dans la conception platonicienne de l'amour, mais notamment dans la scène du débat littéraire de la fin du premier livre. Comme chez Platon, la discussion se déroule au cours de la promenade.

Les Amours de Psyché et de Cupidon apparaissent ainsi comme la synthèse de diverses influences, un «brassage intertextuel " (Jeanneret, $1991: 28$ ) et, avec ces emprunts disparates, se situent à la frontière des genres littéraires. Qualifiés par certains historiens et critiques de " roman $»^{6}$, Les Amours sont en fait difficilement qualifiables. La Fontaine lui-même les nomme tantôt conte, tantôt fable, et dans la Préface il avoue avoir du mal à choisir la forme convenable à son ouvrage : « Je ne savais quel caractère choisir : celui de l'histoire est trop simple ; celui du roman n'est pas encore assez orné ; et celui du poème l'est plus qu'il ne faut » (LA:53). Le poète se résout à embrasser plusieurs genres et manières décrire. Devant assortir, comme il l'avoue, du galant, du merveilleux, de l'héroïque, du relevé, du plaisant, il cherche un juste milieu, puisque :

D’employer l'un en un endroit, et l'autre en un autre, il n'est pas permis ; l'uniformité de style est la règle la plus étroite que nous ayons. J'avais donc besoin d'un caractère nouveau, et qui fût mêlé de tous ceux-là : il me le fallait réduire dans un juste tempérament (LA:53-54).

Le mélange de styles et la coexistence déléments hétérogènes sont donc explicitement signalés par La Fontaine. Le texte porte les traits d'un certain amorphisme, si par ce terme on comprend l'absence de forme et de structure déterminées. Il serait

6. Par exemple P. Clarac, H. Coulet, etc. 
peut-être plus judicieux de considérer Les Amours de Psyché et de Cupidon comme une sorte de sylve (silvae $)^{7}$, ouvrage mixte et protéiforme, juxtaposant différents styles et genres littéraires, ou précisément comme le genre du prosimètre. En tant que prosimètre, Les Amours intègrent au cour de la prose 28 pièces poétiques, de forme et de longueur variables, réparties irrégulièrement dans le récit aussi bien du premier que du second niveau de narration.

Les poésies de Psyché sont de formes et de dimensions fort inégales, allant de deux à 132 vers, strophiques ou plus souvent non-strophiques. Quant à la métrique, c'est l'alexandrin qui prédomine sur le décasyllabe et l'octosyllabe, mais aussi nombreuses sont les strophes hétérométriques. L'inventivité de La Fontaine est remarquable : à côté des strophes régulières, comme le sonnet en alexandrin $(6+6$ - la plainte de Psyché, $L A$ : 95), on rencontre des formes fort irrégulières ${ }^{8}$, tel par exemple le premier poème des Amours ( $L A: 61-62$ ), à l'intérieur duquel l'auteur change de métrique : cinq quatrains d'octosyllabes sont suivi d'un huitain hétérométrique (12/8/8/10/8/4/8/4).

$\mathrm{Si}$ l'on veut déterminer le caractère de la relation entre la prose et le vers dans Psyché, on doit réfléchir d'abord sur la nature et la (les) fonction(s) de ces deux modes d'expression. La question ne se ramène pas à la simple opposition - la prose qui assume la fonction narrative et dramatique et le vers - la fonction descriptive et lyrique. Une telle dichotomie était visible dans le prosimètre de Dante, chez La Fontaine la prose et le vers s'infiltrent mutuellement et peuvent assumer chacun des fonctions multiples. Dans différentes occurrences, l'auteur insiste sur la justesse de telle ou telle option. "Mon principal but est toujours de plaire " (LA : 54) - déclare-t-il dans la Préface et c'est à ce commandement suprême que seront subordonnées toutes ses démarches esthétiques. L'emploi du vers doit donc contribuer à la beauté de lécriture et au plaisir de la lecture : « [je] ne prétends pas que mon ouvrage soit accompli : jai tâché seulement de faire en sorte qu'il plût, et que même on y trouvât du solide aussi bien que de l'agréable. C'est pour cela que j'y ai enchâssé des vers en beaucoup d'endroits» $(L A: 56)$.

Les parties versifiées du récit-cadre sont essentiellement descriptives. La promenade des quatre amis qui veulent admirer les « nouveaux embellissements » $(L A: 60)$ de Versailles est le prétexte à de longues descriptions de ces merveilles : lorangerie, la grotte de Thétis (dont la description se déploie sur plus de 130 vers alexandrins (6+6), LA:63-67) ou les jardins du château.

À l'intérieur de l'histoire de Psyché, le poème, qu'il soit extradiégétique ou intradiégétique (selon si c'est le narrateur ou un personnage de l'histoire qui lénonce), sera introduit dans des moments marquants de l'intrigue, lorsque la dignité du sujet ou l'intensité des sentiments le demandent. Les passages en vers introduisent un ton élevé et solennel comme dans l'exorde à l'histoire de Psyché, dans l'hymne à l'Amour ou l'hymne à la Volupté qui termine le récit de Polyphile. Le narrateur recourt

7. «Choses mêlées».

8. Le vers irrégulier est celui qui domine dans les fables. 
à la poésie par exemple dans la harangue de Vénus, dans le discours de l'oracle, dans la plainte de Psyché, dans le discours de la jeune fille aux dieux des enfers, etc. Le vers est toujours particulièrement prisé dans la description, telle par exemple la représentation des merveilles du palais de l'Amour ou le portrait de Cupidon. La description du char de Vénus est précédée d'une réflexion esthétique dans laquelle le narrateur souligne la nécessité de versifier :

Ceci est proprement matière de poésie : il ne siérait guère bien à la prose de décrire une cavalcade de dieux marins : dailleurs je ne pense pas quon pût exprimer avec le langage ordinaire ce que la déesse parut alors.

C'est pourquoi nous dirons en langage rimé

Que l’empire flottant en demeura charmé $[\ldots](L A: 70)$.

Le commentaire du narrateur, de caractère explicatif et métadiscursif, accompagne souvent l'insertion de la poésie. Citons comme exemples la description du royaume des enfers : «Elles [les singularités des enfers] sont tellement étranges que j’ai besoin d'un style extraordinaire pour vous les décrire » $(L A: 201)$, ou la plainte de Psyché : « Dans cette pensée elle leur disait à peu près les choses que je vais vous dire, et les leur disait en vers aussi bien que moi » (LA:95).

Pour ce qui est des modalités d'insertion des passages versifiés au sein de la prose, différents procédés sont perceptibles. Dans la Vita nova, lalternance de prose et de vers est claire et nette, sans confusion et infiltration réciproques. Il y a un équilibre et une complémentarité entre ces deux modes d'expression. Les poésies étant composées au préalable, le récit et les commentaires sont ajoutés a posteriori et chaque pièce poétique est précédée d'une phrase introductive : "javais résolu de faire une ballade... », « je me proposai de l'exprimer dans un sonnet... », etc. Chez La Fontaine, l'insertion du vers est tantôt annoncée par le narrateur, comme dans les exemples cités ci-dessus, tantôt elle se réalise tout naturellement, sans aucun avis de sa part. Le poème "glisse " dans la prose sans rompre la continuité narrative. Par exemple, dans la description des tapisseries du palais de l'Amour, la présentation des deux premières se fait en cinq quatrains décasyllabiques $(4+6)$, pour continuer en prose :

Je me contenterai donc de parler d'une tapisserie relevée d’or, [...] La tenture était composée de six pièces.

Dans la première on voyait un chaos,

Masse confuse, et de qui l'assemblage

Faisait lutter contre lorgueil des flots

Des tourbillons d'une flamme volage.

[...]

Dans la troisième, Cupidon paraissait assis sur un char tiré par des tigres. [...] La quatrième et la cinquième représentaient en d'autres manières la puissance de Cupidon... etc. (LA:83-84). 
Les passages versifiés ne s'appliquent pas uniquement aux descriptions ou aux épanchements lyriques, comme c'est le cas chez Dante. Le vers peut aussi raconter des événements, rapporter le discours d'un personnage ou même le dialogue (par ex. la plainte de Vénus, la sentence de l'oracle, le discours de Psyché au dragon et aux dieux des enfers). Parfois, à l'intérieur d'un poème sopère le passage de la description à la narration (le palais de l'Amour, les enfers), ou l'inverse (le récit de la "trahison " de Psyché qui aboutit à la description de Cupidon). La poésie nest donc pas un simple ornement de la prose, elle se trouve intégralement liée à la narration, on peut dire qu'elle est au service de la narration.

Le récit de l'histoire de Psyché porte tous les traits de l'oralité. Les nombreux dialogues, caractéristiques de la prose de ce temps, créent parfois l'impression de petites scènes de théâtre. En outre, Polyphile coupe souvent le cours de la narration et s'adresse à ses amis-auditeurs, en appelant à leurs émotions, les invoquant comme témoins de ses propres affections : «Représentez-vous une fille ", "Que vous dirai-je davantage ? ", " je ne saurais vous dépeindre ", " comme je crois vous avoir dit », etc. Ces intrusions fréquentes du narrateur jouent le rôle de métadiscours. Outre qu’elles contribuent à renforcer l'impression d’oralité et d'hybridité, ces digressions brisent la forme et semblent détruire l'illusion romanesque, "le récit met à jour les mécanismes de sa fabrication », comme le dit Michel Jeanneret (1991:33). Citons, à titre d'exemple, quelques-unes de ces digressions. La prose et la poésie en sont également parsemées :

Si jétais plus savant en laart de bien écrire,

Je peindrais ce monarque étendant son empire [...] ( $L A: 65)$;

Parmi les airs qui furent chantés, il y en eut un qui plut particulièrement à Psyché. Je vais vous en dire les paroles, que j’ai mises en notre langue au mieux que j’ai pu [...] ( $L A$ : 78-79; suivent deux sizains de décasyllabes);

Avant que de parler du teint,

Je devais vous avoir dépeint,

Pour aller par ordre en l'affaire.

La posture du Dieu. [...] $(L A: 114)$;

Je vous conterais ses aventures si je ne métais point prescrit des bornes plus resserrées (LA : 164), etc.

Puisque Polyphile est le narrateur qu'on est en droit d'assimiler à l'auteur, le " Je » de Polyphile peut être perçu comme le « Je » de La Fontaine. C'est donc aux activités d'écriture de celui-ci que se rapportent ces digressions métatextuelles.

Outre ces digressions, outre la Préface, qui joue proprement le rôle de paratexte, La Fontaine en tant que poète et écrivain s’exprime le plus nettement dans la scène 
du débat des quatre amis qui termine le premier livre des Amours et qui porte les traits d'un « dialogue lettré », comme l'appelle Claire Cazanave (2005). La discussion, qui se mue en une vraie dispute entre Gélaste et Ariste, porte sur le goût, sur l'esthétique du rire et l'esthétique des larmes, sur la primauté que l'on donne à la tragédie ou à la comédie qui représentent respectivement le sublime et le médiocre, sur le plaisir qui en découle. La question est de savoir ce qui est plus apte à nous toucher - la pitié que suscite la tragédie ou le badinage de la comédie. La controverse demeure irrésolue, peut-être suffit-il de suivre ce précepte d'Ariste selon lequel : " il est bon de saccommoder à son sujet; mais il est encore meilleur de saccommoder à son génie " $(L A: 118)$. Avec ces longues dissertations sur l'esthétique, le texte de La Fontaine, tout comme la Vita nova de Dante, peut être considéré comme une sorte de poétique, une voix importante dans le débat sur les styles et sur les canons du Beau.

Le dernier mot appartiendra à Polyphile qui termine la lecture de son roman par une invocation à la Volupté, éloge épicurien du plaisir et de la diversité. Émile Faguet voit chez La Fontaine un caractère d'épicurien ${ }^{9}$ et Polyphile, porte-parole du poète, est - comme le suggère son nom - celui qui « aimait toutes choses » $(L A: 60)$. Ce nom semble lui convenir à merveille et il dira dans l'hymne à la Volupté :

J'aime le jeu, l'amour, les livres, la musique,

La ville et la campagne, enfin tout; il n'est rien

Qui ne me soit souverain bien,

Jusqu’au sombre plaisir d'un cœur mélancolique $(L A: 220)$.

La Fontaine est le poète de la diversité, il veut chanter sur tous les tons. Dans la discussion des quatre amis, le narrateur semble s’excuser de ce " défaut » : «J'ai déjà mêlé malgré moi de la gaieté parmi les endroits les plus sérieux de cette histoire ; je ne vous assure pas que tantôt je n’en mêle aussi parmi les plus tristes. C’est un défaut dont je ne me saurais corriger, quelque peine que j'y apporte " $(L A: 118)$. Au cœur de lépoque classique, ce siècle féru de pureté et de régularité, La Fontaine propose une œuvre qui s'inscrit plutôt dans l'esthétique baroque de la variété.

Les Amours sont une œuvre qui unit différents tons et manières, comme les amis du récit-cadre représentent chacun un penchant différent : Acante - le lyrique; Ariste - le sérieux ; Gélaste - le gai. C'est en Polyphile - La Fontaine - que sopère la synthèse de toutes ces diverses tendances. Leur fusion fait naitre une œuvre hybride, sans doute, mais quand même équilibrée et achevée, œuvre qui marie harmonieusement la prose et la poésie, le plaisant et le sublime, le récit et le dialogue, qui y enchaîne habilement un ample énoncé métatextuel.

9. Cf. http://gallica.bnf.fr/ark:/12148/bpt6k2023577 


\section{BIBLIOGRAPHIE}

Cazanave C. 2005. Le dialogue au XVII e siècle en France : un genre français moderne ? Élements pour une mise au point. Dix-septième siècle 228. 427-441. URL : https://www. cairn.info/revue-dix-septieme-siecle-2005-3-page-427.htm, consulté le 30/09/2016.

Clarac P. 1969. Littérature française. Lâge classique. II. Paris. Arthaud.

Clarac P. 1972. La Fontaine. Paris. Hatier.

Coulet H. 1967. Le roman jusquả la Révolution. Paris. Armand Colin.

Faguet É. 1913. La Fontaine. URL : http://gallica.bnf.fr/ark:/12148/bpt6k2023577, consulté le $30 / 09 / 2016$.

Jeanneret M. 1991. Introduction. In Les Amours de Psyché et de Cupidon, Jean de La Fontaine. Paris. Livre de Poche. 5-42.

Joubert J.-L. 1988. La poésie. Paris. Armand Colin.

La Fontaine J. de 1991. Les Amours de Psyché et de Cupidon. Édition critique de Michel Jeanneret. Paris. Livre de Poche.

Rousset J. 1976. L'intérieur et l'extérieur. Paris. Librairie José Corti.

\section{The loves of Psyche and Cupid by Jean de La Fontaine as an example of prosi- metrum}

ABSTRACT: The loves of Psyche and Cupid by La Fontaine is a romance of mixed genres, combining the features of a tale, a narrative poem and a philosophical dialogue. The author of this study classifies it as a prosimetrum, to focus her analysis, on the one hand - upon the interrelation between prose and verse, functions and means of introducing rhyming parts, etc., on the other hand - examining metatextual texts of the narrator who provokes interesting aesthetic reflection.

Keywords: La Fontaine, prosimetrum, metatextuality. 